to be an advantageous strategy. Of course, vaccination rates are restricted not only by antigen supply but also by the availability of vaccinators and clinical supplies, such as syringes. Nevertheless, antigen supply remains the greatest restriction for vaccines against COVID-19, and strategies that make the most of available antigen supply would maximize the number of lives saved by vaccination. More generally, in future pandemics, researchers and clinicians involved in vaccine development should consider identifying the appropriate vaccine dosage that can save the most lives for a limited amount of antigen, rather than just a dosage that balances efficacy and reactogenicity for the individual person.
${ }^{1}$ World Health Organization Collaborating Centre for Infectious Disease Epidemiology and Control, School of Public Health, Li Ka Shing Faculty of Medicine, The University of Hong Kong, Hong Kong Special Administrative Region, Hong Kong, China. ${ }^{2}$ Laboratory of Data Discovery for Health Limited, Hong Kong Science and Technology Park, New Territories, Hong Kong Special Administrative Region, Hong Kong, China. ${ }^{3}$ Department of Ecology and Evolution, University of Chicago, Chicago,

IL, USA.

$\bigotimes_{e-m a i l: b c o w l i n g @ h k u . h k}$

Published online: 5 July 2021

https://doi.org/10.1038/s41591-021-01440-4

References

1. Wu, J. T., Peak, C. M., Leung, G. M. \& Lipsitch, M. Lancet 388, 2904-2911 (2016)

2. World Health Organization. Vaccine 35, 5751-5752 (2017).

3. World Health Organization. Wkly. Epidemiol. Rec. 33, 365-380 (2019)
4. Sahin U. et al. Preprint at medRxiv https://doi.org/ 10.1101/2020.12.09.20245175 (2020)

5. Jackson, L. A. et al. N. Engl. J. Med. 383, 1920-1931 (2020).

6. Khoury, D.S. et al. Nat. Med. https://doi.org/10.1038/s41591-021 01377-8 (2021).

7. Voysey, M. et al. Lancet 397, 99-111 (2021).

8. Cobey, S., Larremore, D. B., Grad, Y. H. \& Lipsitch, M. Nat. Rev. Immunol. 21, 330-335 (2021).

\section{Acknowledgements}

B.J.C. is supported by a fellowship award from the Research Grants Council of the Hong Kong Special Administrative Region (Project No. HKU SRFS2021-7S03).

\section{Author contributions}

B.J.C. conceived of this Comment; B.J.C. wrote the original draft of the manuscript with contributions from all authors; and all authors reviewed and approved the final version of the manuscript.

\section{Competing interests}

B.J.C. has consulted for GlaxoSmithKline, Sanofi Pasteur, AstraZeneca, Roche and Moderna.

\title{
Has SARS-CoV-2 reached peak fitness?
}

To the Editor-For the first time in the history of medicine, the evolution of a virus can be observed 'as it happens'. Throughout this, molecular tools of unprecedented accuracy are also assisting by providing an extraordinarily detailed picture of the interactions between the virus and the immune system of its human host.

In the initial period of the COVID-19 pandemic, the coronavirus SARS-CoV-2 used the plasticity of its genome to obvious advantage, increasing its transmissibility with the early appearance of the D614G mutation that led to a $20 \%$ increased infectiousness and that rapidly became the global dominant strain ${ }^{1}$. The next major jump in transmissibility appeared in late 2020 with the B.1.1.7 variant, which had an approximate $50 \%$ increase in transmissibility beyond that of $\mathrm{D} 614 \mathrm{G}^{2}$. The B.1.617.2 'India' variant has recently become prominent in many countries, and although the magnitude is uncertain, it represents another incremental increase in contagiousness beyond that of D614G and possibly that of B.1.1.7 ${ }^{3}$.

In parallel, there has been evolution of variants with properties that allow them to evade the immune system. Thus far, the B.1.351 variant has been demonstrated to have the greatest ability to escape the immune system, although not at a level that has substantially diminished the protection provided by vaccines.
The P.1 variant also has considerable ability to evade the immune response. Although the immunoevasion of D614G and B.1.1.7 is fairly modest, it appears that B.1.617.2 has some features that combine enhanced transmissibility and immunoevasion. Some of the recent enhancement in the ability to escape the immune system is 'tagged' to mutations that alter the $\mathrm{N}$-terminal domain rather than the spike protein's receptor-binding domain, where the early, functionally important alterations were prevalent ${ }^{3}$.

This progression of variants demonstrates the virus's drive for heightened fitness, the natural selection of mutations and strains that make it more likely to find hosts and are further facilitated by sidestepping the immune response, even allowing repeat infections of people who previously had COVID-19. We emphasize, however, that enhanced transmissibility, rather than immunoevasion or greater lethality, would be considered the most potent path for the virus to become more fit and viable.

Indeed, more-fit variants can be expected to emerge over time (the occurrence of which will need to be monitored meticulously, as these pose a potential public health threat), but we believe that these will not continue to emerge indefinitely: nothing is infinite in nature, and eventually the virus will reach its form of 'maximum transmission'. After then, new variants will provide no further advantage in infectivity. The virus will thus stabilize and this 'final' variant will prevail and become the dominant strain, experiencing only occasional, minimal variations.

By homology, we can imagine that the same took place when some very contagious RNA viruses (e.g., measles virus) spilled over in humans: in the early stages of the epidemic, the virus was probably unstable and less transmissible than it is now; then-once the most contagious phenotype was reached-the measles virus stabilized. We note that the inevitable outcome of this strategy for all RNA viruses that have developed high contagiousness (beyond measles virus, we could name, for instance, the viral agents that cause hepatitis A, poliomyelitis, mumps and rubella) is the lack of molecular structures that allow the virus to 'dodge' the immune response of the recovered host. Why is that? To make a long story short, by the time these viruses are attacked by the adaptive immunity of their new host, they have no immediate advantage in evading it, because they have probably already spread to another susceptible host, where replication and survival are ensured ${ }^{4}$.

Notably, the natural history of the SARS-CoV-2 virus-host interplay is now being deeply altered-an unprecedented 
event in a pandemic - by the prompt availability of vaccines that are not only safe but also extremely effective in eliciting a 'superhuman' protective immunity: on the one hand, vaccine-induced immunity is more potent than that elicited by the infection ${ }^{5}$. On the other hand, the most-potent vaccines seem to dramatically hinder viral replication and thus transmission ${ }^{6}$. Because of this, a considerable advantage for the virus could therefore be provided by mutations that cause the emergence of variants that evade vaccine-induced immunity. These would still replicate in the vaccinated host in communities in which the proportion of vaccinated people is very high (e.g., Israel and the United States). However, despite vigorous replication of the virus in these highly vaccinated communities, the world is yet to witness the emergence of variants able to 'break through' the vaccine-induced immunity ${ }^{7}$. We cannot, of course, exclude the possibility of their emergence even in the near future, but molecular data gathered thus far have made it clear that the 'evolutionary space' that SARS-CoV-2 has for evading vaccine-induced immunity is remarkably narrow relative to that available for increases in transmission rates. Some evolutionary virologists believe complete or nearly complete resistance to the current vaccines is an inevitability ${ }^{8}$, which is a prediction that cannot be discounted or ignored.

Therefore, while the world needs to remain on full alert in order to promptly detect the emergence of 'vaccine-piercing' variants and, in that case, rapidly update the available vaccines, there is reason for very cautious optimism. In any case, what is certain is that the emergence of such vaccine-evading variants, if at all possible, will be made more likely by viral spread and replication. May this serve as a reminder that, once infection rates have been controlled in the most advanced countries, it will be crucial to do the same in the developing ones and achieve the same success in more-difficult 'socio-sanitary' settings.

For this reason, it will be in humanity's own interest to not be greedy and instead provide vaccines at an affordable price to all mankind: history has in fact taught that when dealing with the global control of an infectious disease, the most effective way to be selfish is to be generous.

\section{Roberto Burioni ${ }^{1 凶}$ and Eric J. Topol(D) $2 \bowtie$}

${ }^{1}$ Università Vita-Salute San Raffaele, Milano,

Italy. ${ }^{2}$ The Scripps Research Institute, La Jolla,

CA, USA.

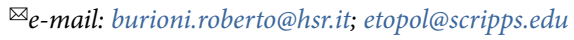

Published online: 21 June 2021

https://doi.org/10.1038/s41591-021-01421-7

References

1. Zhou, B. et al. Nature 592, 122-127 (2021).

2. Washington, N. L. et al. Cell 184, 2587-2594 (2021).

3. Boehm, E. et al. Clin. Microbiol. Infect. https://doi.org/10.1016/ j.cmi.2021.05.022 (2021)

4. Burton, D. R. Nat. Rev. Immunol. 2, 706-713 (2002).

5. Burton, D. R. \& Topol, E. J. Nat. Med. 27, 5-6 (2021).

6. Dagan, N. et al. N. Engl. J. Med. 384, 1412-1423 (2021).

7. Abu-Raddad, L.J. et al. N. Engl. J. Med. https://doi.org/10.1056 NEJMc2104974 (2021).

8. Harvey, T.W. et al. Nat. Rev. Microbiol. 19, 409-424 (2021).

\section{Author contributions}

The concept was developed jointly by R.B. and E.T.; R.B. wrote the first draft, and E.T. revised it.

\section{Choosing Wisely for COVID-19: ten evidence- based recommendations for patients and physicians}

To the Editor-The COVID-19 pandemic has produced devastating effects worldwide, with the causative coronavirus SARS-CoV-2 infecting over 170 million patients and causing more than 3.5 million deaths as of 1 June 2021. The resultant fear and anxiety among the public and treating physicians has frequently resulted in rapid changes to clinical practices and hospital triage decisions, many of which are not evidence based and are often detrimental. There have been wide variations at global and regional levels on guidance related to mask usage, testing protocols, vaccination and patient triage (both hospital admission and escalation to an intensive care unit), as well as the use of various pharmaceutical interventions in treating patients with COVID-19. Many of these deviations from evidence-based healthcare result in substantial harm, as they divert efforts and resources from outcome-based, data-driven best practices toward those that are of doubtful efficacy and are even harmful. As many countries continue to experience repeated waves of COVID-19, it is important to identify practical approaches that are evidence based and implementable in the real world to optimize the use of resources and improve outcomes. Although these are important all over the world, they are crucial in low- and middle-income countries, where resources are scarce.

The Choosing Wisely initiative was begun to promote conversations between patients and physicians about avoiding unnecessary medical interventions. The mission of Choosing Wisely is to help patients and physicians choose care that is evidence based, not duplicative, free from harm, and truly necessary ${ }^{1}$. It works by creating lists of 'things clinicians and patients should question', something that is particularly appropriate to public-health responses and management decisions in the current pandemic. In response to the widespread use of non-evidence-based practices, we initiated Choosing Wisely for COVID-19 to identify 'best buys' for the general public, patients and physicians.

We created an 18-member Choosing Wisely for COVID-19 Task Force in April 2021 with multi-stakeholder involvement. Members were drawn from the fields of public health, epidemiology, general practice, primary care, infectious disease, virology, critical care, internal medicine, pulmonology, pediatrics, oncology, health economics, clinical research, implementation science, and health policy. We also had patient and civil-society representatives to ensure that their views and voices were given due importance. Advisors 